\title{
Robust Optimization-based Calculation of Invariant Trajectory Representations for Point and Rigid-body Motion
}

\author{
Maxim Vochten, Tinne De Laet, and Joris De Schutter
}

\begin{abstract}
Invariant representations of demonstrated motion trajectories provide context-independent motion models that can be used in motion recognition and generalization applications such as robot programming by demonstration. In practice, the use of invariant representations is still limited because their numerical calculation from a demonstrated trajectory is complicated by sensitivity to measurement noise and singularities, yielding inaccurate invariant functions that do not correspond well with the original trajectory. This paper improves the calculation of invariant representations for point and rigid-body motions by reformulating their calculation as an optimization problem that minimizes the error between the trajectory reconstructed from the invariant representation and the measured trajectory. Robustness against noise and singularities is ensured through the addition of regularization terms on the invariants. Simulations and real motion experiments show that the accuracy of the calculated invariant representations greatly improves with respect to standard smoothing methods. These results encourage future developments of motion recognition and generalization applications based on invariant trajectory representations.
\end{abstract}

\section{INTRODUCTION}

Programming by demonstration and human intent recognition rely on motion models that are constructed from the measured trajectory coordinates of demonstrated motions. These motion models are expected to be generally valid and therefore robust against contextual variation such as the arbitrary choice of reference frame in which the coordinates are expressed, the choice of reference points on the tracked object, the location in space where the motion is performed, or the velocity profile and scale with which the motion is executed. In human intent recognition, the motion models need to be general enough to successfully recognize the same motion in different contexts. In programming by demonstration, the models need to be general enough so that motions can be easily applied in different contexts.

The standard approach to generalize motion models is to perform multiple demonstrations of the same motion in different contexts, so that every context is accounted for in the motion model. Gathering this large number of demonstrations is time-consuming however and may require expert knowledge. In that case, it can be helpful to describe the motion using an invariant trajectory representation that is context-independent.

An invariant representation captures intrinsic features from the trajectory that remain unchanged under specific

M. Vochten and J. De Schutter are with the Department of Mechanical Engineering, KU Leuven \& Flanders Make, 3001 Leuven, Belgium. (e-mail: maxim.vochten@kuleuven.be; joris.deschutter@kuleuven.be).

T. De Laet is with the Faculty of Engineering Science, KU Leuven, 3001 Leuven, Belgium. (e-mail: tinne.delaet@kuleuven.be). sets of transformations on the trajectory, such as rotations, translations, and scaling. Many possible invariant representations exist, for an overview see [1]. Here, the focus is on differential invariant representations, hereafter referred to as the invariants, which have several useful properties for model building. These invariants describe a trajectory with functions that capture local differential-geometric properties of the trajectory, also known as signature curves [2]. This local nature is helpful for trajectory recognition [3] since for partially occluded motions, the invariants can still be calculated for the visible parts of the motion. Additionally, the invariants are complete or generative, meaning that the trajectory can be fully generated from the invariants [4]. This is an important property when motions need to be generalized in programming by demonstration.

The primary problems when using differential invariants in applications are the noise sensitivity of their numerical calculation and the existence of singularities. The noise sensitivity is caused by the need for higher-order derivatives of the trajectory coordinates (velocity, acceleration, and jerk) in the analytical formulas of the invariants, resulting in an amplification of noise. Singularities refer to certain points along the trajectory where some of the invariants are undefined, e.g. motion along a straight line. During numerical calculation, these singular points typically lead to high peaks in the invariant signature. Noise sensitivity and singularities in the calculation of the invariants can result in an inaccurate representation of the trajectory. This means that when reconstructing the original trajectory from the invariant signature, large trajectory errors may occur.

Current numerical approaches for calculating invariants primarily focus on combating the noise sensitivity. All approaches typically employ a trajectory filtering or smoothing method in a preprocessing step to reduce noise and calculate the higher-order derivatives of the trajectory coordinates in a numerically stable way, such as the Kalman smoothing method in [3], the spline smoothing method in [5], or the anisotropic smoothing method in [6]. These approaches subsequently calculate the desired invariants using their explicit analytical formulas. Other approaches such as in [2], [7], [8], [9] do not use the analytical formulas of the invariants directly after trajectory smoothing, but instead produce a finite difference approximation of the invariant functions, based on the smoothed coordinates of a set of samples around the considered trajectory point. Reducing the sampling distance to zero yields the analytical formulas again, so that conceptually the idea is similar as in [3], [5], [6]. Singularities are usually ignored by assuming that the 
continuity of the trajectory is high enough. Alternatively, they are handled using ad hoc approaches that rely on an accurate detection of the singular point so that it is either removed or designated as a segmentation point [7]. Some singularities can be resolved by a transformation of the invariants and the moving frame in which they are defined (e.g. the Bishop approach [10]), hereby however losing their local character.

While current approaches starting from the analytical formulas often prove successful in mitigating the effect of noise, the problems of singularities and trajectory errors are usually not solved. This paper's approach in contrast builds on top of the generative property of the invariants. Instead of following the analytical formulas that determine the invariants as a function of the trajectory coordinates, we will ask ourselves the inverse question: "what invariants generate the given measured trajectory?". This becomes possible by reformulating the invariant calculation as an optimal control problem, where the differential invariants are regarded as the control inputs in a dynamical system that governs the generation of the trajectory.

The major contribution of this paper is a novel optimization-based method for calculating differential invariant trajectory representations for two types of motions: point and rigid-body motion. Errors on the trajectory are reduced by a cost term that minimizes the error of the reconstructed trajectory with respect to the measured trajectory. Regularization terms are added on the values and changes of the invariants to mitigate the effect of measurement noise and achieve a smoothing effect on the trajectory. The regularization terms also deal with singularities by driving undefined invariants to zero.

Section II gives mathematical preliminaries on differential invariant representations for point and rigid-body motion. The formulation of the optimal control problem for invariant calculation follows in Section III. Simulations (Section IV) and real motion experiments (Section V) show the effectiveness of the proposed method for dealing with measurement noise and singularities compared to a baseline finite differences method and a Kalman smoothing method. Finally, conclusions are given in Section VI.

\section{INVARIANT TRAJECTORY REPRESENTATIONS}

This section reviews the definition of the invariant representations for point motion (Frenet-Serret invariants [11]) and rigid-body motion (instantaneous screw axis invariants [12]). The problems of singularities and noise sensitivity are illustrated for the Frenet-Serret invariants.

A common property of the discussed invariant representations is that they are defined and expressed in a moving frame, that progresses along with the trajectory. Reconstructing the trajectory from the invariants first requires reconstructing the motion of the moving frame from the invariants. Then, the object trajectory can be reconstructed using the location of the moving frame and the invariants. This process is captured in a set of generative equations that are provided in this section and which form the foundation of the proposed optimization method for calculating invariants.

\section{A. Point motion - Frenet-Serret invariants}

The motion of a point in space can be characterized by three invariant functions: $i_{1}, i_{2}$, and $i_{3}$, defined and expressed as a function of time $t$ in a local moving frame, referred to as the Frenet-Serret frame $\{F S\}$. The first invariant $i_{1}$ is defined along the first axis of $\{F S\}$ and corresponds to the speed with which the object moves along its path. The second and third invariant $i_{2}$ and $i_{3}$ describe the motion of $\{F S\}$ itself. They are rotational velocities along the second and first axis of $\{F S\}$. The three Frenet-Serret invariants [3] of a point motion trajectory are analytically defined by the translational velocity vector $\boldsymbol{v}(t)$ of the point and its firstand second-order time-derivatives $\dot{\boldsymbol{v}}(t)$ and $\ddot{\boldsymbol{v}}(t)$ :

$$
\begin{gathered}
i_{1}(t)=\|\mathbf{v}(t)\| ; \quad i_{2}(t)=\frac{\|\mathbf{v}(t) \times \dot{\mathbf{v}}(t)\|}{\|\mathbf{v}(t)\|^{2}} \\
i_{3}(t)=\frac{([\mathbf{v}(t) \times \dot{\mathbf{v}}(t)] \cdot \ddot{\mathbf{v}}(t))\|\mathbf{v}(t)\|}{\|\mathbf{v}(t) \times \dot{\mathbf{v}}(t)\|^{2}}
\end{gathered}
$$

Optionally, the invariants can be made time-independent and therefore purely geometric by using the arc length as progress parameter along the trajectory instead of time.

Reconstruction of the trajectory first requires the orientation of the Frenet-Serret frame $\{F S\}$ at each time. The orientation of $\{F S\}$ with respect to a reference frame can be expressed through the definition of the rotation matrix

${ }^{F S} \boldsymbol{R}$. The time-evolution of the orientation ${ }^{F S} \dot{\boldsymbol{R}}(t)$ is then given as a function of the Frenet-Serret invariants as follows:

$$
{ }^{F S} \dot{\boldsymbol{R}}(t)={ }^{F S} \boldsymbol{R}(t)\left[\begin{array}{ccc}
0 & 0 & i_{2}(t) \\
0 & 0 & -i_{3}(t) \\
-i_{2}(t) & i_{3}(t) & 0
\end{array}\right] .
$$

These equations are also known as the Frenet-Serret differential equations [11], though note we use a different order of the axes to be consistent with other invariant representations. Integration of these dynamics equations starting from the initial frame orientation ${ }^{F S} \boldsymbol{R}(0)$ results in the location of $\{F S\}$ at every time instant. Knowing the location of $\{F S\}$, the motion trajectory of the point ${ }^{o b j} \boldsymbol{p}(t)$ is obtained by integrating the following equation:

$$
{ }^{o b j} \dot{\boldsymbol{p}}(t)={ }^{F S} \boldsymbol{R}(t)\left(\begin{array}{c}
i_{1}(t) \\
0 \\
0
\end{array}\right),
$$

given the initial location of the point ${ }^{o b j} \boldsymbol{p}(0)$. This corresponds to the integration of the velocity vector along the tangent to the point curve.

Singularities are points along the trajectory where some of the invariants are undefined. For the Frenet-Serret invariants this occurs when the point is standing still $(\|\mathbf{v}(t)\|=0)$ or when the point is moving along a straight line $(\| \mathbf{v}(t) \times$ $\dot{\mathbf{v}}(t) \|=0$ ). These cases are illustrated below in Section II-C.

\section{B. Rigid-body motion - instantaneous screw axis invariants}

The Frenet-Serret invariants describe changes in the point motion trajectory, expressed in a local moving frame $\{\mathrm{FS}\}$ based on the translational velocity vector $\boldsymbol{v}$ and its derivatives. For rigid-body motions, a generalization of this concept 
can be made through Chasles' theorem, which states that the instantaneous motion of a rigid body can be represented by a rotation and translation along some axis in space referred to as the Instantaneous Screw Axis (ISA). In [12], this theorem is used to describe rigid-body motion trajectories by six invariant functions $\omega_{1}, \omega_{2}, \omega_{3}, v_{1}, v_{2}$, and $v_{3}$, defined and expressed in a local moving frame $\{i s a\}$ that is attached to the ISA. Two invariants $\omega_{1}$ and $v_{1}$ describe the rotation and translation of the rigid body along the ISA (which lies along the first axis of $\{i s a\}$ ). The other four invariants $\omega_{2}, \omega_{3}, v_{2}$, and $v_{3}$ describe the motion of the moving frame $\{i s a\}$, where $\omega_{2}$ and $\omega_{3}$ are rotational velocities along the second and first axis of $\{i s a\}$ respectively, while $v_{2}$ and $v_{3}$ give the translation of the origin of the frame along the same axes. Analytical formulas for the ISA invariants are given in [12].

Reconstructing the rigid-body trajectory from the invariants requires the pose (i.e. position and orientation) of the moving frame $\{i s a\}$. The pose of $\{i s a\}$ and the rigid-body trajectory $\{o b j\}$ are denoted by the homogeneous transformation matrix ${ }^{a} \boldsymbol{T}$, that defines the pose of a frame $\{a\}$ with respect to the reference frame. It extends the rotation matrix ${ }^{a} \boldsymbol{R}$ containing the orientation of frame $\{a\}$ with a position vector ${ }^{a} \boldsymbol{p}$ representing the position of the origin of $\{a\}$ with respect to the given reference frame: ${ }^{a} \boldsymbol{T}=\left[\begin{array}{cc}{ }^{a} \boldsymbol{R} & { }^{a} \boldsymbol{p} \\ 0_{1 \times 3} & 1\end{array}\right]$.

The time-evolution of the pose of $\{i s a\},{ }^{i s a} \dot{\boldsymbol{T}}(t)$, is defined by the four invariants $\omega_{2}, \omega_{3}, v_{2}$, and $v_{3}$ as follows:

$$
{ }^{i s a} \dot{\boldsymbol{T}}(t)={ }^{i s a} \boldsymbol{T}(t)\left[\begin{array}{cccc}
0 & 0 & \omega_{2}(t) & v_{3}(t) \\
0 & 0 & -\omega_{3}(t) & v_{2}(t) \\
-\omega_{2}(t) & \omega_{3}(t) & 0 & 0 \\
0 & 0 & 0 & 0
\end{array}\right]
$$

Integration of these dynamics equations from the initial pose ${ }^{i s a} \boldsymbol{T}(0)$ gives the $\{i s a\}$ frame at every time instant $t$.

The motion trajectory of the rigid body ${ }^{o b j} \boldsymbol{T}$ is obtained using the location of $\{i s a\}$ and the two remaining invariants $\omega_{1}$ and $v_{1}$ by integrating the following equation:

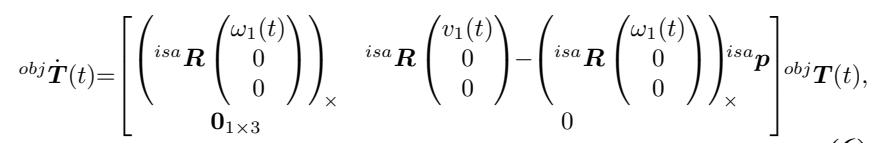

where $(\cdot)_{\times}$denotes the $3 \times 3$ skew-symmetric matrix form of a $3 \times 1$ vector. Given the initial pose of the rigid body ${ }^{o b j} \boldsymbol{T}(0)$, the complete trajectory can be reconstructed.

Singularities for the instantaneous screw axis invariants are discussed in detail in [12]. They occur when the rigid body is either undergoing a pure translation (i.e. no rotation, $\left.\omega_{1}=0\right)$ or when the screw axis remains parallel to itself (rotation in the plane, $\omega_{2}=0$ ).

\section{Illustration of singularities and noise sensitivity}

The problems of singularities and sensitivity to measurement noise are illustrated for the Frenet-Serret invariants.

As explained before, singularities occur when some of the Frenet-Serret invariants are undefined. A first type of singularity occurs when the magnitude of the velocity $\|\mathbf{v}\|$ is zero, i.e. at a stationary point (Figure 1a). From the analytical

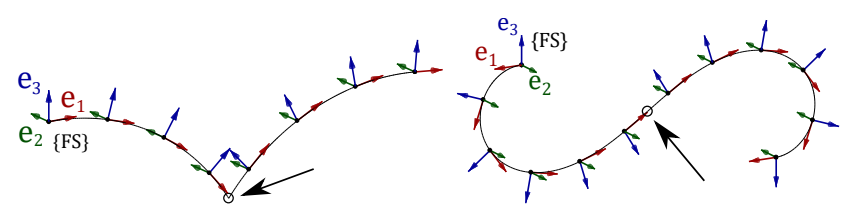

(a) Stationary singularity

(b) Inflection singularity

Fig. 1. Illustration of singularities in the definition of the Frenet-Serret invariants for point trajectories. The axes of the Frenet-Serret frame $\{F S\}$ moving along with the point trajectory are visualized. In the singularities, part of the invariants is undefined resulting in a discontinuity in the motion of the moving frame.

definition of the invariants in (1) and (2), it is clear that $i_{2}$ and $i_{3}$ become undefined. Since the Frenet-Serret frame, moving along the trajectory, is related to the values of the invariants, it is also undefined and exhibits a discontinuity in its motion. This discontinuity corresponds to large peaks in the invariant signature. A second type of singularity occurs when $\|\mathbf{v} \times \dot{\mathbf{v}}\|=0$, i.e. when the curve locally becomes a straight line. If the curve changes from convex to concave or vice versa, it is referred to as an inflection, otherwise it is known as an undulation. In the case of an inflection (Figure 1b), the Frenet-Serret frame flips about the tangent vector corresponding to a large peak in the third invariant $i_{3}$. Singular points often appear in practice and the numerical calculation of the invariants in these points consequently leads to noise-sensitive peaks in the invariant signature, which is undesirable for recognition based on invariants.

The noise sensitivity of the invariants is caused by the need for higher-order derivatives of the trajectory coordinates in the analytical formulas (1) and (2). This becomes clear by calculating the invariants using derivatives obtained with a simple forward finite differences method. Figure $2 b$ shows the devastating result of calculating the invariants for a discretely sampled curve $(100 \mathrm{~Hz})$ using finite differences where only a small amount of Gaussian noise $(\sigma=0.05 \mathrm{~mm})$ was added to the trajectory coordinates. As a result, the measurement noise is greatly amplified in the invariants.

Both the influence of singularities and the sensitivity to noise lead to errors in the calculation of the invariants. When reconstructing trajectories from the invariants using equations (3) and (4), this inevitably leads to integration errors where the shape of the trajectory may be severely distorted. Figure 2a shows the influence of noisy invariants on the reconstructed trajectory. This is problematic in applications where the trajectory needs to be accurately reproduced.

\section{OPTIMIZATION-BASED INVARIANTS CALCULATION}

The previous section showed how the invariants can be calculated explicitly through analytical formulas that contain the derivatives of the trajectory coordinates. Generative equations provided the means to reconstruct the original trajectory from the invariants. This section proposes an alternative calculation approach for the invariants by embedding these generative equations in an Optimal Control Problem (OCP) formulation, where the goal is to find the invariants that produce a given measured trajectory. An optimal control 


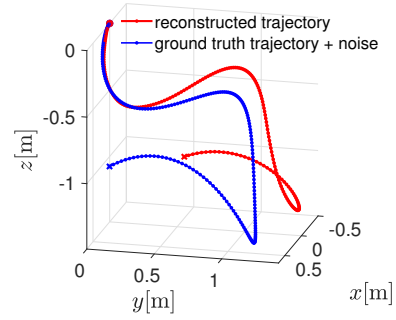

(a) Point trajectory
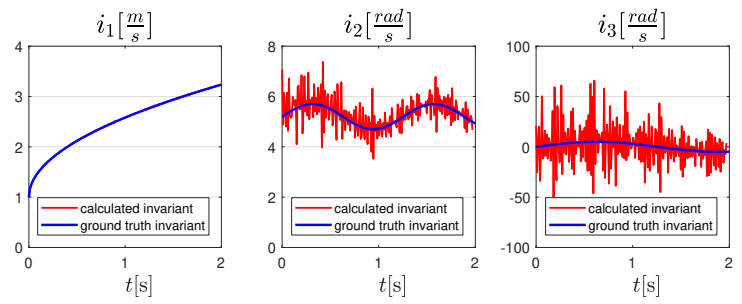

(b) Frenet-Serret invariant

Fig. 2. Illustration of noise sensitivity when calculating the FrenetSerret invariants of a discretely sampled point trajectory $(100 \mathrm{~Hz})$ using finite differences. After adding a small amount of noise $(\sigma=0.05 \mathrm{~mm})$ on the trajectory, the calculated invariants show a large amplification of measurement noise. Reconstructing the trajectory from the calculated invariants consequently leads to large trajectory errors.

problem is an optimization problem that finds an optimal choice of control inputs to drive the states of a dynamical system to a desired result. It is specified by a cost or objective function and a set of constraints on the states and controls. The relation between states and controls is given by a dynamical system $\dot{\boldsymbol{x}}(t)=\boldsymbol{f}(\boldsymbol{x}, \boldsymbol{u}, t)$ that is added in the constraints, specifying the evolution of the states $\boldsymbol{x}$ as a function of the control inputs $\boldsymbol{u}$.

For point motion trajectories, the dynamical system corresponds to the Frenet-Serret differential equations (3)-(4). The controls $\boldsymbol{u}$ correspond to the unknown Frenet-Serret (FS) invariants $\boldsymbol{u}=\left(i_{1}, i_{2}, i_{3}\right)$, while the system states $\boldsymbol{x}$ consist of the Frenet-Serret frame orientation and the position of the point along the trajectory, $\boldsymbol{x}=\left({ }^{F S} \boldsymbol{R},{ }^{o b j} \boldsymbol{p}\right)$.

For rigid-body motion trajectories, the dynamical system analogously corresponds to the differential equations in (5)(6). The controls $\boldsymbol{u}$ correspond to the instantaneous screw axis (ISA) invariants $\boldsymbol{u}=\left(\omega_{1}, \omega_{2}, \omega_{3}, v_{1}, v_{2}, v_{3}\right)$, while the system states $\boldsymbol{x}$ consist of the pose of the $\{i s a\}$ frame and object frame $\{o b j\}$ along the trajectory, $\boldsymbol{x}=\left({ }^{i s a} \boldsymbol{T},{ }^{o b j} \boldsymbol{T}\right)$.

The rest of this section first discusses the choice of cost terms in the objective function, then formulates the optimization problem in continuous time, and finally discretizes the optimization problem using a multiple shooting approach together with a receding window method.

\section{A. Cost terms}

The objective function of the optimization problem is designed such that the problems of trajectory errors, noise sensitivity, and singularities are dealt with simultaneously.

1) Trajectory accuracy cost: The first cost function expresses the accuracy with which the generated trajectory from the invariants should correspond to the trajectory measurements. For point trajectories, it is defined as the deviation between the generated position ${ }^{o b j} \boldsymbol{p}$ and measured position ${ }^{o b j} \boldsymbol{p}_{m}$ at a certain time $t$ :

$$
\Delta \boldsymbol{p}_{\text {des }}(t)={ }^{o b j} \boldsymbol{p}(t)-{ }^{o b j} \boldsymbol{p}_{m}(t) .
$$

Perfect correspondence between the generated trajectory and measurements means that $\Delta \boldsymbol{p}_{\text {des }}(t)=\mathbf{0}$ at every time $t$.

For rigid-body trajectories, the accuracy cost is defined as the error between the generated pose ${ }^{\text {obj }} \boldsymbol{T}(t)$ and the measured pose ${ }^{o b j} \boldsymbol{T}_{m}$ of the rigid body:

$$
\Delta \boldsymbol{T}_{\text {des }}(t)={ }^{o b j} \boldsymbol{T}(t)^{-1{ }^{o b j}} \boldsymbol{T}_{m}(t) .
$$

Perfect correspondence between the generated trajectory and measurements means that $\Delta \boldsymbol{T}_{d e s}(t)=\boldsymbol{I}_{4}$ at every time $t$.

In the optimization problem, this cost will be integrated over the time horizon of the motion so that the entire trajectory integration error is taken into account.

2) Smoothing regularization cost: To reduce noise sensitivity, a regularization cost $\boldsymbol{r}(t)$ is added that consists of the time-derivative of the invariants (notation: " ' "). For point trajectories (FS invariants) it is equal to:

$$
\boldsymbol{r}(t)=\left(\begin{array}{lll}
i_{1}^{\prime}(t) & i_{2}^{\prime}(t) & i_{3}^{\prime}(t)
\end{array}\right)^{T},
$$

while for rigid-body trajectories (ISA invariants) it is:

$$
\boldsymbol{r}(t)=\left(\begin{array}{llllll}
\omega_{1}^{\prime}(t) & \omega_{2}^{\prime}(t) & \omega_{3}^{\prime}(t) & v_{1}^{\prime}(t) & v_{2}^{\prime}(t) & v_{3}^{\prime}(t)
\end{array}\right)^{T} .
$$

The physical interpretation of the term $\boldsymbol{r}(t)$ is a constraint on the acceleration of the moving point or rigid body and on the acceleration of the corresponding moving frame.

3) Singularity regularization cost: In singularities, the invariants are undefined and could therefore take an arbitrary value. A regularization cost term $s(t)$ is added that forces the invariants to zero. For point motion trajectories (FS invariants) the singularity regularization cost is:

$$
\boldsymbol{s}(t)=\left(i_{2}(t) \quad i_{3}(t)\right)^{T},
$$

while for rigid-body trajectories (ISA invariants) it is:

$$
\boldsymbol{s}(t)=\left(\begin{array}{llll}
\omega_{2}(t) & \omega_{3}(t) & v_{2}(t) & v_{3}(t)
\end{array}\right)^{T} .
$$

The effect of forcing the invariant values to zero also has the beneficial effect of limiting unnecessary motion of the corresponding moving frames.

\section{B. Continuous optimal control problem formulation}

The optimal control problem for calculating the FrenetSerret invariants $F S \mathbf{i}$ of point trajectories is now formulated as follows using the cost functions defined above:

$$
\begin{aligned}
& F S \mathbf{i}=\operatorname{argmin} \int_{0}^{T}\left\|\Delta \boldsymbol{p}_{\text {des }}(t)\right\|_{W_{1}}^{2}+\|\boldsymbol{r}(t)\|_{W_{2}}^{2}+\|\boldsymbol{s}(t)\|_{W_{3}}^{2} \mathrm{~d} t \\
& \text { subject to } \quad F S \text { dynamics equations (3)-(4) } \\
& F S \boldsymbol{R}(0)^{F S} \boldsymbol{R}(0)^{T}-\boldsymbol{I}_{3}=0 .
\end{aligned}
$$

Analogously, the instantaneous screw axis invariants ${ }^{i s a} \mathbf{i}$ for rigid-body trajectories are found as: 
${ }^{i s a} \mathbf{i}=\operatorname{argmin} \int_{0}^{T}\left\|\Delta \boldsymbol{T}_{\text {des }}(t)-\boldsymbol{I}_{4}\right\|_{W_{1}}^{2}+\|\boldsymbol{r}(t)\|_{W_{2}}^{2}+\|\boldsymbol{s}(t)\|_{W_{3}}^{2} \mathrm{~d} t$

subject to ISA dynamics equations (5)-(6)

$$
\begin{aligned}
& { }^{i s a} \boldsymbol{R}(0)^{i s a} \boldsymbol{R}(0)^{T}-\boldsymbol{I}_{3}=0 \\
& { }^{o b j} \boldsymbol{R}(0)^{o b j} \boldsymbol{R}(0)^{T}-\boldsymbol{I}_{3}=0 .
\end{aligned}
$$

The objective function consists of the three cost terms defined above, integrated over the whole time horizon and weighted using weighting matrices $W_{1}, W_{2}$, and $W_{3}$. The weighting matrices could be constant or time-varying. For $\left\|\Delta T_{\text {des }}-I_{4}\right\|_{W_{1}}$, the Frobenius norm is used with weight $w_{r}$ for the rotation elements and $w_{p}$ for the position.

The constraints include the dynamics equations (3)-(4) or (5)(6) that relate the evolution of the trajectory with the invariant control inputs. To ensure the orientation matrices $\boldsymbol{R}$ in the state dynamics remain orthogonal, orthonormality constraints (15), (18), (19) need to be introduced in at least one time step. Here the first time step is chosen. The orthogonality will be preserved throughout the whole time horizon thanks to the state dynamics equations.

\section{Discretized optimal control problem formulation}

Discretization is a necessary step before the optimal control problem (OCP) for calculating invariants can be applied to discretely sampled trajectory measurements. The OCP is discretized with a direct multiple shooting method [13]. The time horizon is partitioned in $N$ intervals: $j=\{0,1, \ldots, N\}$, where the controls are considered piecewise constant over these intervals and the states are variables on the discrete interval points. This transforms the integrals in the objective functions (13) and (16) to sums.

For the state dynamics of the form $\dot{\boldsymbol{x}}(t)=\boldsymbol{f}(\boldsymbol{x}, \boldsymbol{u}, t)$, an integrator function $\boldsymbol{F}$ is defined so that the dynamics equations are transformed into the following equality constraints: $\boldsymbol{x}_{j+1}-\boldsymbol{F}\left(\boldsymbol{x}_{j}, \boldsymbol{u}_{j}\right)=0$. To integrate the orientation dynamics involving the rotation matrix $\boldsymbol{R}$, a custom integrator is needed to ensure that the orthogonal properties are preserved. Orientation dynamics of the following form:

$$
\dot{\boldsymbol{R}}(t)=\boldsymbol{R}(t)[\boldsymbol{\omega}(t)]_{\times},
$$

are exactly integrated using exponential mapping [14]:

$$
\boldsymbol{R}_{j+1}=\boldsymbol{R}_{j} \exp \left(\left[\boldsymbol{\omega}_{j}\right]_{\times} \Delta T\right),
$$

with $\omega_{j}$ considered constant over the integration interval $\Delta T$. For example, when integrating the orientation of the Frenet-Serret frame ${ }^{F S} \boldsymbol{R}, \boldsymbol{\omega}_{j}$ corresponds to the invariants $\left[\begin{array}{lll}i_{3} & i_{2} & 0\end{array}\right]^{T}$. Something analogous happens for the rotation matrices ${ }^{i s a} \boldsymbol{R}$ and ${ }^{o b j} \boldsymbol{R}$ in the pose matrices ${ }^{i s a} \boldsymbol{T}$ and ${ }^{{ }^{b j}} \boldsymbol{T}$.

Solving the optimization problem can become computationally expensive for a large number of samples $N$ if the time horizon $T$ is long and/or the time step $\Delta T$ is short. Furthermore, in on-line applications such as human intent prediction, it is necessary to calculate invariants while the motion is still being executed by the human. Therefore, we implement a receding window approach, where instead of

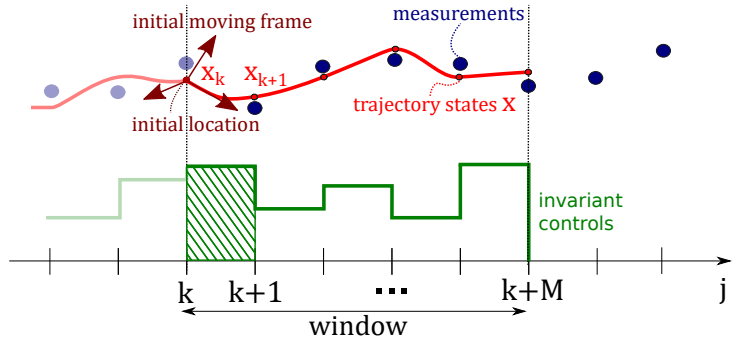

Fig. 3. Receding window approach. For a given initial trajectory state $\mathbf{x}_{k}$, the invariants are calculated in a window of $M$ samples. Afterwards, only the first invariant control is applied to the trajectory to reach $\mathbf{x}_{k+1}$. The window then moves one sample to the right, and the process is repeated.

solving one large optimization over all samples $N$, a series of small optimizations is solved in a window with size $M<<N$ that progresses over the samples.

The receding window approach is sketched in Figure 3. Starting from a fixed initial state $\boldsymbol{x}_{k}$ of the trajectory at the first sample $k$, the invariants along with the corresponding trajectory (and moving frames) are calculated in a window $[k, k+M]$ given the available trajectory measurements, which can be considered as reference inputs for the trajectory state. After solving the optimization problem, only the first invariant control input between $k$ and $k+1$ is applied to the system, resulting in a new state estimate $\boldsymbol{x}_{k+1}$ of the trajectory and moving frame at sample $k+1$. Afterwards, the window moves one sample to the right and the whole process repeats itself until the end of the motion is reached. At the start of the motion, the first state $\boldsymbol{x}_{1}$ needs to be chosen. In this paper, the initial object location in $\boldsymbol{x}_{1}$ is set equal to the first measurement, while the initial moving frame is calculated using a finite difference approximation of the first three measurements.

The results of discretizing the continuous OCP using the multiple shooting method and the receding window approach are as follows for the Frenet-Serret invariants $F S \mathbf{i}$ :

$$
\begin{aligned}
F S \mathbf{i}=\operatorname{argmin} & \sum_{j=k+1}^{k+M}\left\|\Delta \boldsymbol{p}_{\text {des }, j}\right\|_{W_{1}}^{2}+\left\|\boldsymbol{r}_{j}\right\|_{W_{2}}^{2}+\left\|\boldsymbol{s}_{j}\right\|_{W_{3}}^{2} \\
\text { subject to } & \text { discrete FS dynamics eqs (3)-(4) } \\
& { }^{F S} \boldsymbol{R}_{k}={ }^{F S} \hat{\boldsymbol{R}}_{k}, \quad{ }^{o b j} \boldsymbol{p}_{k}={ }^{o b j} \hat{\boldsymbol{p}}_{k}, \quad \text { (24) }
\end{aligned}
$$

with ${ }^{F S} \hat{\boldsymbol{R}}_{k}$ and ${ }^{o b j} \hat{\boldsymbol{p}}_{k}$ obtained from the previous window; and for the instantaneous screw axis invariants ${ }^{i s a} \mathbf{i}$ :

$$
\begin{array}{r}
{ }_{i s a} \mathbf{i}=\operatorname{argmin} \sum_{j=k+1}^{k+M}\left\|\Delta \boldsymbol{T}_{\text {des }, j}-\boldsymbol{I}_{4}\right\|_{W_{1}}^{2}+\left\|\boldsymbol{r}_{j}\right\|_{W_{2}}^{2}+\left\|\boldsymbol{s}_{j}\right\|_{W_{3}}^{2} \\
\text { subject to } \quad \begin{array}{l}
\text { discrete ISA dynamics eqs (5)-(6) } \\
\text { isa } \boldsymbol{T}_{k}={ }^{i s a} \hat{\boldsymbol{T}}_{k}, \quad{ }^{o b j} \boldsymbol{T}_{k}={ }^{o b j} \hat{\boldsymbol{T}}_{k}, \quad \text { (26) }
\end{array}
\end{array}
$$

with ${ }^{i s a} \hat{\boldsymbol{T}}_{k}$ and ${ }^{o b j} \hat{\boldsymbol{T}}_{k}$ obtained from the previous window. The explicit orthogonality constraints (15), (18), (19) from the continuous formulation are not necessary anymore, since there is now a hard constraint on the first state $\boldsymbol{x}_{k}$, so that the orthogonal properties of the first state are automatically preserved over the whole horizon through the state dynamics. 
The discretized optimal control problem was implemented using the CasADi software [15] and numerically solved using IPOPT [16].

\section{SYNTHETIC EXPERIMENTS}

The proposed optimization method for calculating invariants is evaluated in simulation to verify robustness against measurement noise and the influence of singularities. The chosen examples are all point motions for which the FrenetSerret (FS) invariants will be calculated. Synthetic measurements of the position together with the corresponding invariants provide a ground truth. Three methods for calculating the FS invariants are compared:

1) Finite differences method (FD): first, the higher-order derivatives of the trajectory coordinates are calculated using forward finite differences on the measured position coordinates. Afterwards, the FS invariants are calculated using the analytical formulas in (1) and (2). This method serves as a baseline.

2) Kalman smoother method (KS): the derivatives of the trajectory coordinates are calculated in a numerically stable way by applying a Kalman smoother to the whole trajectory with a constant derivative-jerk model [3]. The standard deviation on the process noise is set to $1000 \mathrm{~m} / \mathrm{s}^{4}$ and the standard deviation on the measurement noise is set to $0.001 \mathrm{~m}$. Afterwards, the FS invariants are again calculated using the analytical formulas in (1) and (2).

3) Optimization method (OCP): the FS invariants are calculated as the solution of the optimization problem in (22)-(24). The regularization weights in the matrices $W_{1}, W_{2}$, and $W_{3}$ have the following constant values:

$W_{1}=\left[\begin{array}{lll}1 & 0 & 0 \\ 0 & 1 & 0 \\ 0 & 0 & 1\end{array}\right], W_{2}=\left[\begin{array}{ccc}1 e^{-2} & 0 & 0 \\ 0 & 1 e^{-3} & 0 \\ 0 & 0 & 1 e^{-3}\end{array}\right], W_{3}=\left[\begin{array}{cc}1 e^{-10} & 0 \\ 0 & 1 e^{-10}\end{array}\right]$.

The window size is set to 30 samples.

\section{A. Robustness to measurement noise}

The influence of measurement noise is investigated for a helical-like point trajectory (sample rate $50 \mathrm{~Hz}$ ). Its velocity and torsion invariants remain constant at $i_{1}(t)=1 \mathrm{~m} / \mathrm{s}$ and $i_{3}(t)=2 \mathrm{rad} / \mathrm{s}$, while the curvature invariant $i_{2}$ varies:

$$
\begin{cases}i_{2}(t)=8 \frac{\mathrm{rad}}{\mathrm{s}}, & 0 s \leq t<2 s \\ i_{2}(t)=4 \frac{\mathrm{rad}}{\mathrm{s}}, & 2 s \leq t<4 s \\ i_{2}(t)=0.25 \frac{\mathrm{rad}}{\mathrm{s}}, & 4 s \leq t<6 s\end{cases}
$$

Gaussian white noise $\sigma_{m}$ is added to the trajectory for three different noise levels: $\sigma_{m}=0.001 \mathrm{~m}, \sigma_{m}=0.005 \mathrm{~m}$, and $\sigma_{m}=0.01 \mathrm{~m}$. The root-mean-square (RMS) error between the calculated invariants and the ground-truth invariants is computed and averaged over ten simulation runs for each of the three calculation methods. Analogously, the RMS error is calculated between the ground-truth trajectory and the trajectory reconstructed from the invariants.

Table I summarizes all the results. The errors on the first two invariants $i_{1}$ and $i_{2}$ are comparable for the $\mathrm{KS}$ and OCP method, while the errors for the FD method are nearly

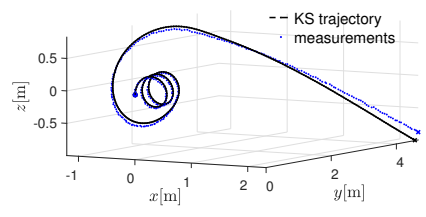

(a) KS trajectory

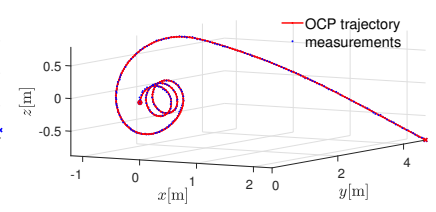

(b) OCP trajectory
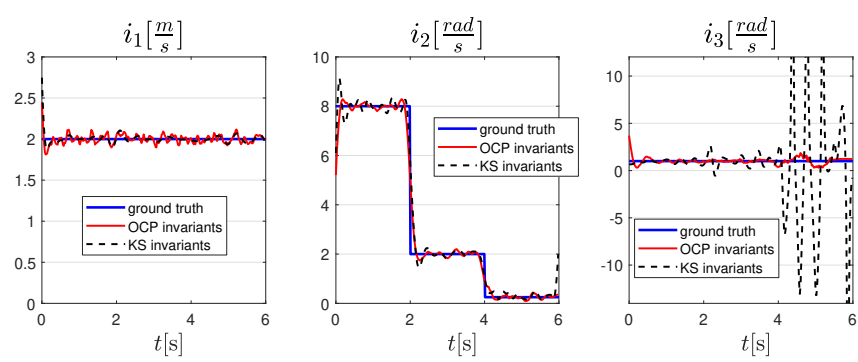

(c) Frenet-Serret invariants

Fig. 4. The invariants are calculated for a noisy $\left(\sigma_{m}=0.005 \mathrm{~m}\right)$ helicallike trajectory using the Kalman smoother method (KS) and the optimization method (OCP). For the KS method, $i_{3}$ becomes more and more inaccurate when nearing the singularity $\left(i_{2}=0\right)$. The trajectory error remains smaller for the OCP method.

always an order of magnitude greater. For $i_{3}$ however, there is an important difference between the KS and OCP method depending on the value of $i_{2}$. Figure 4 shows, for one of the trials, that when $i_{2}$ is close to zero (a singularity), the error on $i_{3}$ drastically increases for the KS method. The same holds for the FD method (not visualized). For the OCP method, proximity to singularities has no influence on the accuracy of the calculated invariants.

The trajectory error remains much lower for the OCP method compared to the FD and KS method since for the latter methods any error in the invariants results in an integration drift on the reconstructed trajectory. Figure 4a shows the growing trajectory error for the KS method. Since the FD method always scores much worse than the other methods, it is strongly advised not to use it.

\section{B. Dealing with singularities}

The effect of singularities in the Frenet-Serret invariants is investigated further in detail for the KS and OCP method with different examples (no added noise). The first example (Figure 5) features a stationary singularity since the velocity $i_{1}$ becomes zero in the middle of the trajectory for 0.8 seconds. The third invariant $i_{3}$ is not visualized here since it is a 2D motion. The OCP approach is capable of dealing with this singularity by driving the second invariant $i_{2}$ to zero. Afterwards, it rises again to the desired value. The rising is able to start ahead of the actual increase thanks to the moving window, which takes later measurements into account. At the trajectory level, the moving frame makes a smooth transition over the discontinuity. For the KS method, the second invariant $i_{2}$ is estimated erroneously in the singularity. This leads to a large trajectory error after the singularity.

Finally, Figure 6 shows an example of an inflection singularity $\left(i_{2}=0\right)$. The ground truth features a positive curvature rate $i_{2}$ and a peak in the torsion rate $i_{3}$ that corresponds 
TABLE I

COMPARISON OF RMS ERRORS ON THE CALCULATED FS INVARIANTS AND RECONSTRUCTED TRAJECTORY OF A HELICAL-LIKE POINT MOTION WITH ADDED MEASUREMENT NOISE $\sigma_{m}$ FOR THREE METHODS: FINITE DIFFERENCES (FD), KALMAN SMOOTHER (KS), AND OPTIMIZATION (OCP).

\begin{tabular}{|c|c|c|c|c|c|c|c|c|c|c|c|c|}
\hline & \multicolumn{3}{|c|}{ trajectory error $\Delta p[\mathrm{~m}]$} & \multicolumn{3}{|c|}{ invariant error $\Delta i_{1}[\mathrm{~m} / \mathrm{s}]$} & \multicolumn{3}{|c|}{ invariant error $\Delta i_{2}[\mathrm{rad} / \mathrm{s}]$} & \multicolumn{3}{|c|}{ invariant error $\Delta i_{3}[\mathrm{rad} / \mathrm{s}]$} \\
\hline & $\mathrm{FD}$ & KS & OCP & FD & KS & $\mathrm{OCP}$ & $\mathrm{FD}$ & KS & $\mathrm{OCP}$ & FD & KS & $\mathrm{OCP}$ \\
\hline & 4.42 & 0.0673 & 0.00408 & 0.0692 & 0.0249 & 0.0235 & 3.32 & 0.386 & 0.439 & 66.7 & 1.08 & 0.166 \\
\hline $5 \mathrm{~m}$ & 2.57 & 0.0975 & 0.00663 & 0.347 & 0.0276 & 0.0323 & 19.6 & 0.411 & 0.465 & 83.6 & 4.55 & 0.613 \\
\hline$\sigma_{m}=0.01 \mathrm{~m}$ & 2.41 & 0.199 & 0.0136 & 0.701 & 0.0461 & 0.0729 & 47.0 & 0.536 & 0.847 & 66.1 & 13.0 & 1.89 \\
\hline
\end{tabular}

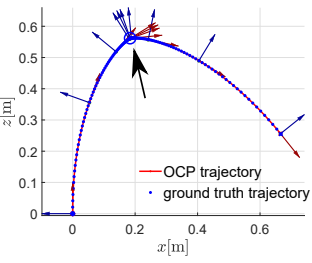

(a) OCP trajectory

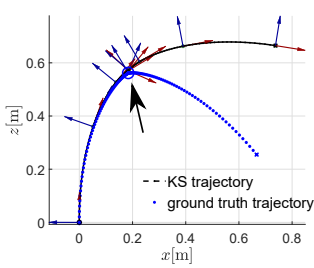

(c) KS trajectory
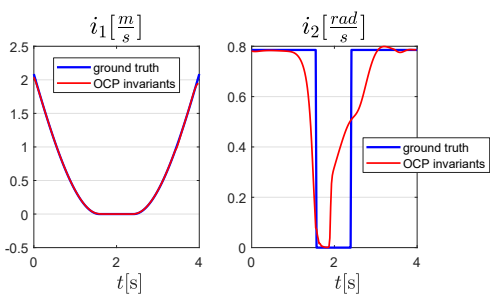

(b) OCP invariants
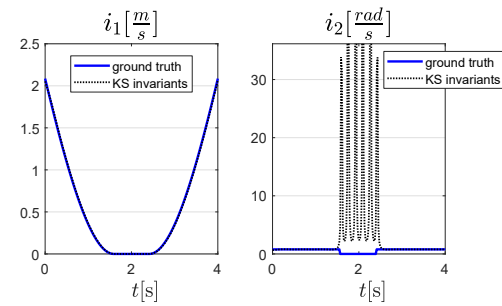

(d) KS invariants
Fig. 5. Example of a stationary singularity $\left(i_{1}=0\right)$ for the optimization method (OCP) and the Kalman smoother method (KS). The OCP method makes a smooth transition of the Frenet-Serret frame over the singularity. The KS method produces large errors for the second invariant $i_{2}$ resulting in a faulty trajectory after the singularity.

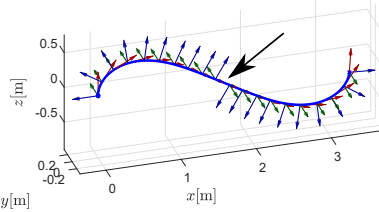

(a) Ground truth trajectory

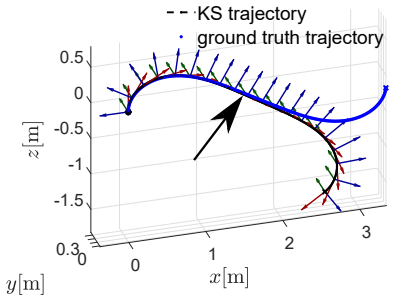

(c) KS trajectory

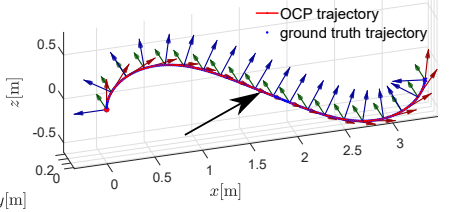

(b) OCP trajectory with $i_{2}$ changing sign

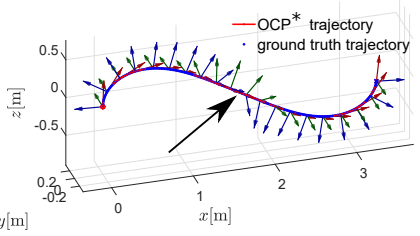

(d) OCP* trajectory with $i_{2}$ positive

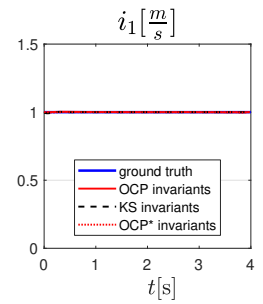

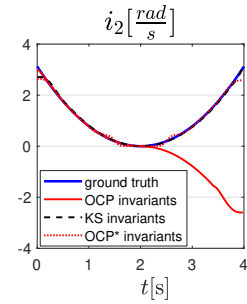

(e) Frenet-Serret invariants

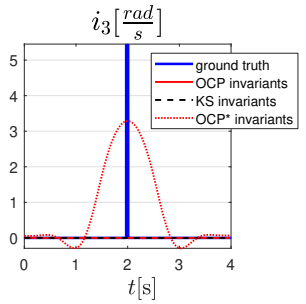

Fig. 6. Example of an inflection singularity $\left(i_{2}=0\right)$. The standard OCP method allows negative values for $i_{2}$ so that the peak in $i_{3}$ is completely avoided. Alternatively with the $\mathrm{OCP}^{*}$ method, where $i_{2}$ is always positive, a smoothing of the peak in $i_{3}$ over multiple samples is achieved. with a rotation of the Frenet-Serret frame around its first axis of $180^{\circ}$. For the OCP method (Figure 6b), the curvature rate $i_{2}$ can be both positive or negative, hereby avoiding the peak in $i_{3}$. This is because the evolution of the Frenet-Serret frame is investigated over multiple samples given an initial orientation, so that it is possible to know whether the curve is locally convex or concave. The discontinuity on the FrenetSerret frame is then completely avoided. Alternatively, if the OCP method is adjusted (OCP* method) such that $i_{2}$ is constrained to be always positive like in the analytical definition, then the resulting effect on the moving frame will be a smooth transition instead of a discontinuous flip. The peak in $i_{3}$ is smoothed over multiple samples. For the KS method, the inflection was not correctly detected so that large trajectory errors occur.

\section{MOTION EXPERIMENTS}

The optimization method for calculating invariants is evaluated with some real demonstrated rigid-body motion trajectories. Since these are rigid-body motions, the instantaneous screw axis (ISA) invariants are calculated instead of the Frenet-Serret invariants for point motion.

Two methods are compared for calculating the ISA invariants: the Kalman smoother method (KS) from Section IV and the optimization method (OCP), where the ISA invariants are calculated as the solution of the optimization problem in (25)-(27). The regularization weights are chosen as: $W_{1}=$ $\left\{w_{r}, w_{p}\right\}=\{0.5,1\}, W_{2}=1 e^{-2} \boldsymbol{I}_{6}, W_{3}=1 e^{-10} \boldsymbol{I}_{4}$, while the receding window length is set to 40 samples.

The rigid-body motions are recorded using the Krypton K600 camera system from NIKON Metrology. The Krypton system tracks the position of LED markers attached to the manipulated object at $50 \mathrm{~Hz}$. If at least three markers are visible at all times, then the pose ${ }^{o b j} \boldsymbol{T}_{m}$ of the object can be fully defined with respect to the reference frame. These pose measurements are used for calculating the invariants.

Three distinct motion classes are considered: hammering, pouring, and shaking. Ten instances of each motion class are executed to introduce some variation. The six ISA invariants are then calculated for each motion instance, using both the $\mathrm{KS}$ and the OCP method. Afterwards, the average standard deviation of each invariant is calculated over all instances of each motion class and over the whole time horizon (after resampling all invariants to 200 samples).

Table II summarizes the average standard deviation on each invariant and for all motion classes. For the lower-order invariants $\omega_{1}$ and $v_{1}$, the standard deviation of the invariants has the same order of magnitude for both the KS and the OCP method. This is because the values of $\omega_{1}$ and $v_{1}$ are not influenced by the presence of singularities and consist only of 
TABLE II

COMPARISON OF THE AVERAGE STANDARD DEVIATION $(s t d)$ ON THE CALCULATED INSTANTANEOUS SCREW AXIS INVARIANTS OF THREE MOTION CLASSES USING TWO CALCULATION METHODS: KALMAN SMOOTHER (KS) AND OPTIMIZATION (OCP).

\begin{tabular}{l|rr||rr||rr} 
& \multicolumn{2}{|c|}{ hammering } & \multicolumn{2}{c||}{ pouring } & \multicolumn{2}{c}{ shaking } \\
std & KS & OCP & KS & OCP & KS & OCP \\
\hline$\omega_{1}[\mathrm{rad} / \mathrm{s}]$ & 0.566 & 0.507 & 0.269 & 0.245 & 0.789 & 0.610 \\
$v_{1}[\mathrm{~m} / \mathrm{s}]$ & 0.0421 & 0.0322 & 0.0168 & 0.0147 & 0.0218 & 0.0329 \\
$\omega_{2}[\mathrm{rad} / \mathrm{s}]$ & 8.66 & 2.59 & 4.69 & 1.04 & 14.6 & 4.91 \\
$v_{2}[\mathrm{~m} / \mathrm{s}]$ & 6.54 & 0.387 & 1.14 & 0.173 & 2.05 & 0.192 \\
$\omega_{3}[\mathrm{rad} / \mathrm{s}]$ & 13.6 & 2.49 & 14.4 & 2.41 & 8.54 & 2.96 \\
$v_{3}[\mathrm{~m} / \mathrm{s}]$ & 41.4 & 1.67 & 20.6 & 0.463 & 14.1 & 0.224
\end{tabular}

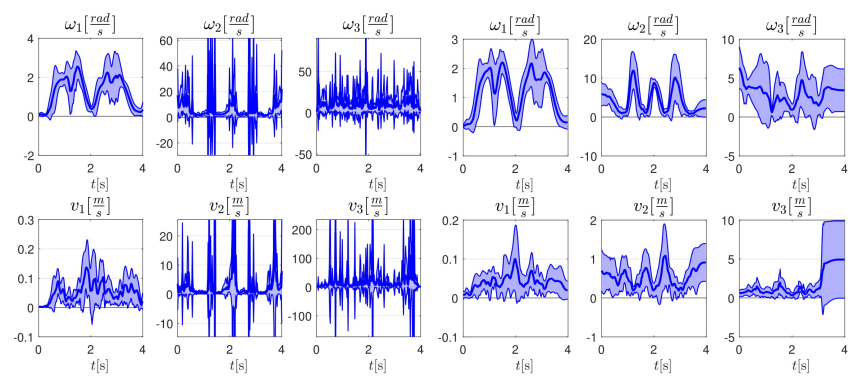

(a) ISA invariants with KS method (b) ISA invariants with OCP method

Fig. 7. Mean and standard deviation of the six instantaneous screw axis invariants (ISA) invariants for the hammering rigid-body motion class using the Kalman smoother method (KS) and the optimization method (OCP).

the first-order derivative of the trajectory coordinates. For the invariants $\omega_{2}, \omega_{3}, v_{2}$, and $v_{3}$, the occurrence of singularities and the noise sensitivity of the higher-order derivatives of the trajectory coordinates result in a much larger standard deviation for the KS method with respect to the invariants obtained by the OCP method. Figure 7 further illustrates these results for the hammering motion class.

\section{CONCLUSIONS}

The objective of this paper was to find a new approach to calculate differential invariant trajectory representations for point and rigid-body motions with attention to the following properties: (1) robustness against measurement noise, (2) correct estimation of invariants in singular points, and (3) correct reproduction of the original trajectory from the invariants. This was achieved by formulating the calculation of the invariants as an optimization problem where the error between the measured trajectory and the reconstructed trajectory from the invariants is minimized. Extra regularization terms in the objective function on the invariants make it capable of dealing with measurement noise and singularities.

Evidently, the solution of the optimization problem depends on the choice of the weights in the optimization problem. The most suitable choice generally depends on the sampling time, the expected noise level, the geometric scale of the motion, and the particular application at hand. Though this could be seen as a disadvantage of the method, note that other methods such as the Kalman smoother method also rely on parameter tuning to weight the influence of noisy data.

Compared to other approaches for calculating invariants, the optimal control method suffers from long calculation times since a nonlinear optimization problem needs to be solved. However, for relatively small window sizes $(\leq 30$ samples), the invariants in the window can be calculated quite fast in approximately 0.05 seconds, even with non-optimized code. The reason is that the receding window is initialized with the solution of the previous one, leading to convergence in only a few (three to five) iterations.

The low standard deviation of the invariants within a motion class suggests that the recognition rate during human motion classification can be improved with respect to current calculation methods using invariant models. On-line recognition is also possible thanks to the fast calculation with the receding window approach.

Errors on the trajectory reconstructed from the invariants remain small, even in the case of measurement noise and singularities. This makes the optimization method suitable for trajectory generalization in programming by demonstration, where it is important that the generated trajectory resembles the original demonstration.

\section{ACKNOWLEDGMENTS}

All authors gratefully acknowledge the financial support by the Flemish FWO project G0B6613. The KU Leuven Robotics Research Group is a core lab of Flanders Make. This work was supported by Flanders Make ICON project FINROP (Fast and Intuitive Robot Programming).

\section{REFERENCES}

[1] I. Weiss, "Geometric invariants and object recognition," International Journal of Computer Vision, vol. 10, no. 3, pp. 207-231, 1993

[2] E. Calabi, P. J. Olver, C. Shakiban, A. Tannenbaum, and S. Haker, "Differential and numerically invariant signature curves applied to object recognition," International Journal of Computer Vision, vol. 26, no. 2, pp. 107-135, Feb 1998

[3] M. Vochten, T. De Laet, and J. De Schutter, "Comparison of rigid body motion trajectory descriptors for motion representation and recognition," in 2015 IEEE International Conference on Robotics and Automation (ICRA), May 2015, pp. 3010-3017.

[4] —_, "Generalizing demonstrated motions and adaptive motion generation using an invariant rigid body trajectory representation," in 2016 IEEE International Conference on Robotics and Automation (ICRA), May 2016, pp. 234-241.

[5] K. R. Kim, P. T. Kim, J. Y. Koo, and M. R. Pierrynowski, "Frenetserret and the estimation of curvature and torsion," IEEE Journal of Selected Topics in Signal Processing, vol. 7, no. 4, pp. 646-654, Aug 2013.

[6] C. Rao, A. Yilmaz, and M. Shah, "View-invariant representation and recognition of actions," International Journal of Computer Vision, vol. 50, no. 2, pp. 203-226, 2002.

[7] S. Wu and Y. F. Li, "Flexible signature descriptions for adaptive motion trajectory representation, perception and recognition," Pattern Recognition, vol. 42, no. 1, pp. 194-214, 2009.

[8] D. Lee, R. Soloperto, and M. Saveriano, "Bidirectional invariant representation of rigid body motions and its application to gesture recognition and reproduction," Autonomous Robots, vol. 42, no. 1, pp. 125-145, Jan. 2018.

[9] T. Moons, E. J. Pauwels, L. J. Van Gool, and A. Oosterlinck, "Foundations of semi-differential invariants," International Journal of Computer Vision, vol. 14, no. 1, pp. 25-47, 1995.

[10] R. L. Bishop, "There is more than one way to frame a curve," The American Mathematical Monthly, vol. 82, no. 3, pp. 246-251, 1975.

[11] W. Kühnel, Differential geometry: curves-surfaces-manifolds. American Mathematical Soc., 2006, vol. 16

[12] J. De Schutter, "Invariant description of rigid body motion trajectories," Journal of Mechanisms and Robotics, vol. 2, no. 1, pp. $011004 / 1-9,2010$.

[13] M. Diehl, H. G. Bock, H. Diedam, and P.-B. Wieber, "Fast direct multiple shooting algorithms for optimal robot control," in Fast motions in biomechanics and robotics. Springer, 2006, pp. 65-93.

[14] R. M. Murray, S. S. Sastry, and L. Zexiang, A Mathematical Introduction to Robotic Manipulation, 1st ed. Boca Raton, FL, USA: CRC Press, Inc., 1994.

[15] J. Andersson, "A General-Purpose Software Framework for Dynamic Optimization," $\mathrm{PhD}$ thesis, Arenberg Doctoral School, KU Leuven, Department of Electrical Engineering (ESAT/SCD) and Optimization in Engineering Center, 3001-Heverlee, Belgium, October 2013.

[16] A. Wä̀chter and L. T. Biegler, "On the implementation of an interiorpoint filter line-search algorithm for large-scale nonlinear programming," Mathematical Programming, vol. 106, no. 1, pp. 25-57, 2006. 